\title{
Influence of Metallic lons on the Aggregation of Soft Colloidal Polysaccharides
}

\author{
Jean-Pierre Sagou Sagou ${ }^{1, *}$, Séka Simplice Kouassi ${ }^{2}$, Fabien Thomas ${ }^{3}$ \\ ${ }^{1}$ Laboratory of Inorganic Materials Chemistry, Felix Houphouet BOIGNY University, Abidjan, Ivory Coast \\ ${ }^{2}$ Laboratory of Environmental and Materials Chemistry, Jean Lorougnon GUEDE University, Daloa, Ivory Coast \\ ${ }^{3}$ Interdisciplinary Laboratory for Continental Environments, Lorraine University, Vandoeuvre lès Nancy, France
}

\section{Email address:}

jsagou@yahoo.fr (Jean-Pierre S. S.), sekasimplice@yahoo.fr (Séka S. K.), fabien.thomas@univ-lorraine.fr (Fabien T.)

${ }^{*}$ Corresponding author

\section{To cite this article:}

Jean-Pierre Sagou Sagou, Séka Simplice Kouassi, Fabien Thomas. Influence of Metallic Ions on the Aggregation of Soft Colloidal Polysaccharides. Science Journal of Chemistry. Vol. 5, No. 2, 2017, pp. 23-30. doi: 10.11648/j.sjc.20170502.12

Received: March 20, 2017; Accepted: March 30, 2017; Published: April 24, 2017

\begin{abstract}
The aggregation behavior of carboxymethyldextran, a soft and anionic polysaccharide, was studied in the presence of divalent cation $\mathrm{Cd}^{2+}$ in aqueous $\mathrm{NaNO}_{3}$ electrolyte. The experimental destabilization conditions of this colloidal suspension have determined by turbidimetric method and the kinetics of aggregation and the characteristics of aggregates were analyzed through Dynamic Light Scattering measurements. The aggregates are derived from very attractive interactions, which are modulated by the metallic ion to carboxymethylextran ratios and their feature indicates a mechanism characterized by a diffusion-limited aggregation.
\end{abstract}

Keywords: Carboxymethyldextran, Soft Particle, Interactions, Turbidity, Aggregation

\section{Introduction}

Aggregation is a process in which particles join together to form larger structures called aggregates or flocs. Aggregation in colloidal dispersions, suspensions and emulsions, is important for many natural phenomena and industrial processes [1, 2], as solar cells [3, 4] energy storage [5] electronics [6] optics [7]. The subject of aggregation has received considerable attention [8-16] to get a fundamental and quantitative understanding of its mechanism. The existence of two limiting and universal aggregation modes has so been established and recognized by several experimental [17-26] and theoretical [27-34] studies: the diffusion-limited aggregation (DLA) [35-37] and the reaction-limited aggregation (RLA) [38-40]. The difference between them is how the particles stack together. DLA is rapid, being limited solely by the diffusion time between collisions. Each collision between two approaching clusters or particles leads to an irreversible sticking, resulting in loosely connected hyperbranched clusters with a typical fractal dimension of 1.7-1.8. The average mass of the clusters is a linear function of the aggregation time. In
RLA, only a very small fraction of collisions can result in one sticking. This aggregation mechanism is slower, since in this case, a repulsive barrier exists between the clusters. Consequently, a large number of collisions is required before two particles can stack together. Thus, one approaching cluster or particle has a much higher chance to interpenetrate the surface of an existing large cluster before it is finally stuck on it, resulting in a denser cluster with a fractal dimension of 2.0-2.2, depending on its polydispersity. The average mass of the clusters grows exponentially with the aggregation time. In macromolecular systems, the aggregation is often induced by physical, chemical or biological changes in the molecular state, which are due to their flexible secondary and tertiary structure formed by intra- and intermolecular bonds [41]. In addition, this coagulation is modulated by the concentration, nature of the solvent, nature of the substituents, central metal ions and temperature [42]. With respect to rigid particles, the aggregation of soft particles has been less frequently studied. So far, only a few 
experimental studies on soft colloidal aggregation have been reported in the literature [21-24, 43]. The present paper is devoted to an acquisition of experimental data for a synthesized soft material. To that end, we have analyzed the kinetics of aggregation and the characteristics of the aggregates formed in aqueous solutions of a soft colloidal polysaccharide, the carboxymethyldextran (CMD) in presence of a metallic ion, the cadmium ion $\left(\mathrm{Cd}^{2+}\right)$. This study has consisted of two parts. The first step allowed to define turbidimetrically the experimental destabilization conditions of the $\mathrm{CMD} /$ metal system and the second one has been carried out in order to understand the aggregation mechanism and describe the features of the clusters and the kinetics of their evolution in time by using the dynamic light scattering technique.

\section{Experimental Section}

\subsection{Experimental Material}

The chemicals listed below were from analytical grades and were used as received: $\mathrm{NaNO}_{3}$ and $\mathrm{Ca}\left(\mathrm{NO}_{3}\right)_{2}$ from Prolabo (Merck, USA); $\mathrm{HNO}_{3}, \mathrm{NaOH}, \mathrm{ClCH}_{2} \mathrm{COOH}$ and $\left\{\mathrm{Cd}(\mathrm{NO} 3)_{2}, 4 \mathrm{H}_{2} \mathrm{O}\right\}$ from Aldrich (St. Quentin Fallavier,
France). Ultrapure Milli-Q water was used to prepare polymer solutions. Carboxymethyldextran was obtained by carboxymethylation of native Dextran T500 purchased from Amersham Biosciences (Uppsala, Sweden) with monochloroacetic acid under alkaline conditions as detailed elsewhere [44-46].

\subsection{Material Characterization}

The degree of substitution (DS), i.e. the amount of carboxymethyl groups per 100 glucopyranosidic units, and the volume charge density $(\rho 0 / \mathrm{F})$ of the synthesized CMD are determined by potentiometric titration at $100 \mathrm{mM} \mathrm{NaNO}_{3}$ ionic strength and $\mathrm{pH} 7$ (complete dissociation of the carboxylic sites). The hydrodynamic diameter (D) of the synthesized CMD and native Dextran T500, and their weight average molar mass $\mathrm{Mw}$ were measured at $25.0 \pm 0.1 \mathrm{C}$ using a Zetasizer Nano ZS instrument (He-Ne red laser $(633 \mathrm{~nm})$, Malvern Instruments) and the multi angle laser light scattering coupled to steric exclusion chromatography (SECMALLS), respectively. Table 1 lists several properties of the native Dextran T500 and the functionalized carboxymethyldextran.

Table 1. Values of the degree of substitution (DS), volume charge density ( $\left.q_{0} / F\right)$, hydrodynamic diameter (D) and weight-average molar mass (Mw) of the Native Dextran T500 and CMD at pH 7 in $100 \mathrm{mM} \mathrm{NaNO}$.

\begin{tabular}{lllll}
\hline Polymer & DS (\%) & $\mathbf{q}_{\mathbf{0}} / \mathbf{F}(\mathbf{m o l} / \mathbf{m} 3)$ & D $(\mathbf{n m})$ & Mw $(\mathbf{g} / \mathbf{m o l})$ \\
\hline Dextran T500 & - & - & 40 & 634,800 \\
CMD & 49 & 38 & 58 & 794,000 \\
\hline
\end{tabular}

\subsection{Sample Preparation}

Initial Polysaccharide solutions were prepared $24 \mathrm{~h}$ prior to experiments in $\mathrm{NaNO}_{3}$ electrolyte solution and stored at $4{ }^{\circ} \mathrm{C}$ to ensure the complete dispersion of polymers. Batch experiments were carried out by adding variable amounts of metal concentration solution to a given volume of polysaccharide solution. It should be pointed out that the procedure for the preparation of the aggregating systems is crucial to measure correctly the aggregation kinetics. To that end, the metal is added to macromolecular solution without mixing action and followed for aggregation experiment. In contrast, prior to turbidimetric measurements, the polysaccharide/metal mixing was stirred for 2 hours and kept at rest for 17 hours in order to provide information about the formation of soluble or insoluble complexes trough colloidal dispersion.

\subsection{Methods}

\subsubsection{Turbidity Measurements}

Turbidity measurements were performed with a $2100 \mathrm{~N}$ Hach Turbidimeter equipped with tungsten filament as the light source. As turbidity values are lower than 40, only one detector located at $90^{\circ}$ to the light source carries out the measurement. Above this value, all detectors are used and the turbidity is an average of measurements coming from the three sensors based on the scattered light/transmitted light ratio. For each value of the metal to polymer concentrations ratio examined, several independent suspensions were prepared and runs were performed to ensure the reproducibility of the results. All measurements were carried out at $\mathrm{pH} 7$ and at $25 \pm 1^{\circ} \mathrm{C}$.

\subsubsection{Dynamic Light Scattering Measurements}

Aggregation kinetics of carboxymethyldextran were followed by Photon Correlation Spectroscopy technique using a Malvern Instruments Ltd. PCS100 Series 4700 spectrometer. This equipment is constituted of goniometer mounted on a rotating arm. A laser beam of $514 \mathrm{~nm}$ is used as the light source and directed to the sample contained in a quartz cell, which is maintained in the VAT, a calibrated cuvette, implanted in the rotation axis of the spectrometer. A diaphragm controls the amount of scattered light detected and sends it back to the photomultiplier which is sensitive to the quantity of individual photons and allows to fix a diffusion angle ranged from $10^{\circ}$ to $150^{\circ}$. Data acquisition were made from a correlator which converts the signal whose processing is carried out by a suitable software "CONTIN" provided by Malvern. Measurements were carried out at $90^{\circ}$ relative to the incident beam at $\mathrm{pH} 7$ and $25 \pm 1{ }^{\circ} \mathrm{C}$. 


\section{Results and Discussion}

\subsection{Analysis of CMD Destabilization Conditions}

In this investigation, three total CMD concentrations have been studied: $0.5 \mathrm{~g} / \mathrm{L}, 1 \mathrm{~g} / \mathrm{L}$ and $2 \mathrm{~g} / \mathrm{L}$. The amount of the polymer concentration $(\mathrm{P})$ and the metal concentration (M) are given by the molar concentration ratios $(\mathrm{M} / \mathrm{P})$. The metal to polymer concentration ratio corresponds to the situation in which the polymer concentration is fixed and variable amounts of metal concentration solution are added to polymer solution. In order to reduce the complexity of the problem as connected to swelling and segment distribution heterogeneity [46-48], the analyses are carried out for CMD suspensions in $100 \mathrm{mM} \mathrm{NaNO}_{3}$ electrolyte solution. In the first step of our study, we analysed the turbidimetry of CMD macromolecules in presence of variable concentrations of divalent cations $\mathrm{Ca}^{2+}$ and $\mathrm{Cd}^{2+}$.

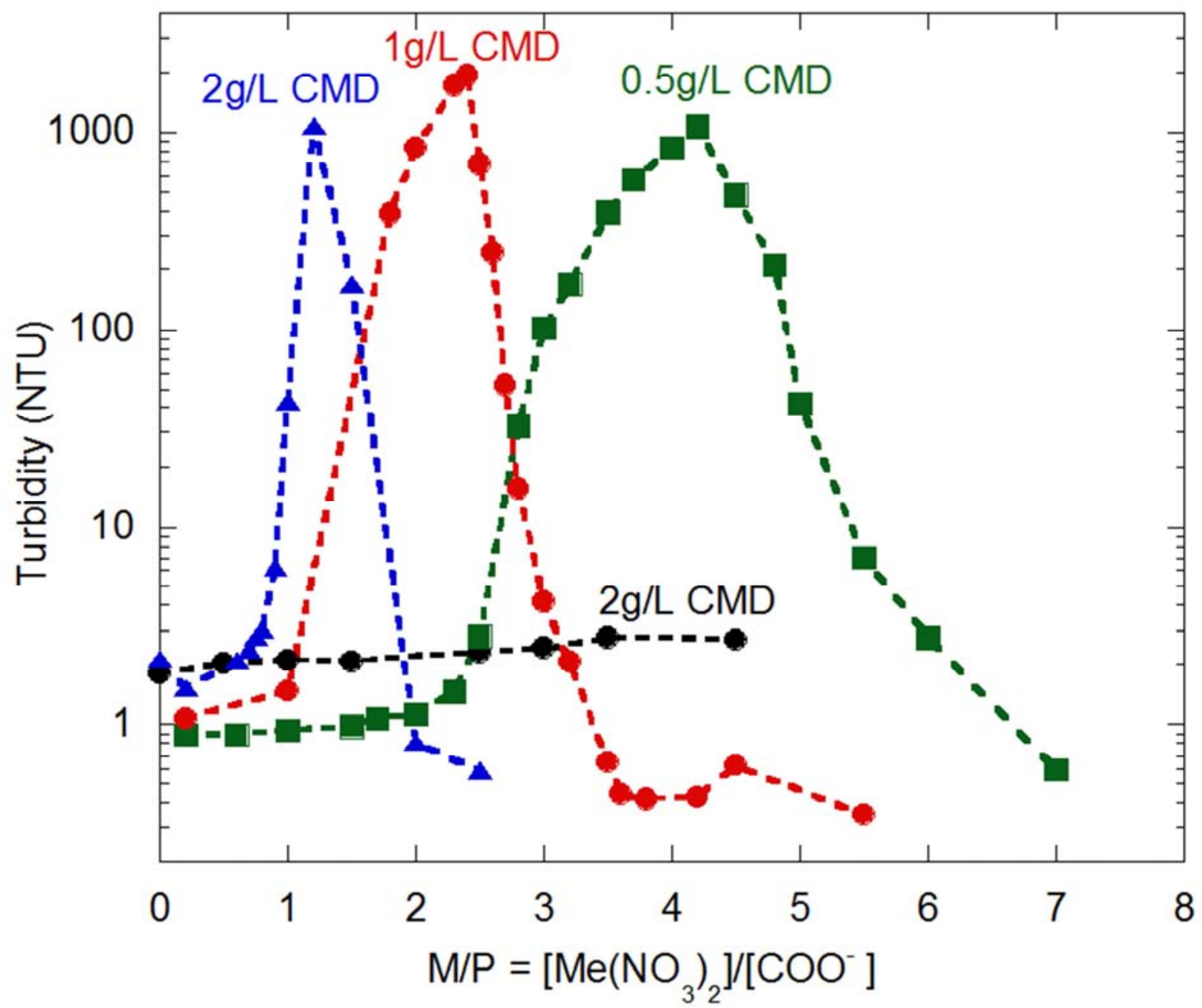

Figure 1. Turbidity of $C M D$ as a function of the metal to polymer ratio $(M / P)$ in presence of $C d^{2+}$ for $0.5 \mathrm{~g} / \mathrm{L} C M D(\bullet), 1 \mathrm{~g} / \mathrm{L} C M D(\bullet), 2 \mathrm{~g} / \mathrm{L} C M D(\mathbf{\Delta})$ and in presence of $\mathrm{Ca}^{2+}$ for $2 \mathrm{~g} / \mathrm{L} C M D(\bullet)$.

Figure 1 exhibits the evolution of this turbidity as a function of the metal/polymer concentrations ratios $(\mathrm{M} / \mathrm{P})$, which shows that the shape of plots depends on the metal type with which the polyion interacts. In presence of $\mathrm{Ca}^{2+}$, the turbidity is almost constant and close to that of the CMD solution without metal $(\mathrm{M} / \mathrm{P}=0)$, suggesting that the interactions between calcium and carboxylate ions are no sufficiently high to modify the turbidity even for higher metal/polymer ratios. The photo (Figure 2 (B)) corresponding to one of that polymer/calcium mixtures shows a suspension completely transparent as reference solution (Figure 2 (A)). This result evidences weak interactions between $\mathrm{Ca}^{2+}$ and CMD at supramolecular scale, already previously highlighted through the study at molecular level [49]. Unlike $\mathrm{Ca}^{2+}$, the situation is different for $\mathrm{Cd}^{2+}$ for which the plots of turbidity for the three tested CMD concentrations show a large dependence on the $\mathrm{M} / \mathrm{P}$ ratios. Even if plots are shift towards lower values of metal/polymer ratios as polymer concentration increased, the plots present similar general shapes. Firstly, the turbidity sharply increases up to a maximum with an increase of metal/polymer ratio to a certain dosage. Then, the turbidity decreases and at last for sufficiently large metal/polymer ratios, it becomes lower than the turbidity of CMD macromolecules suspension without metal. 


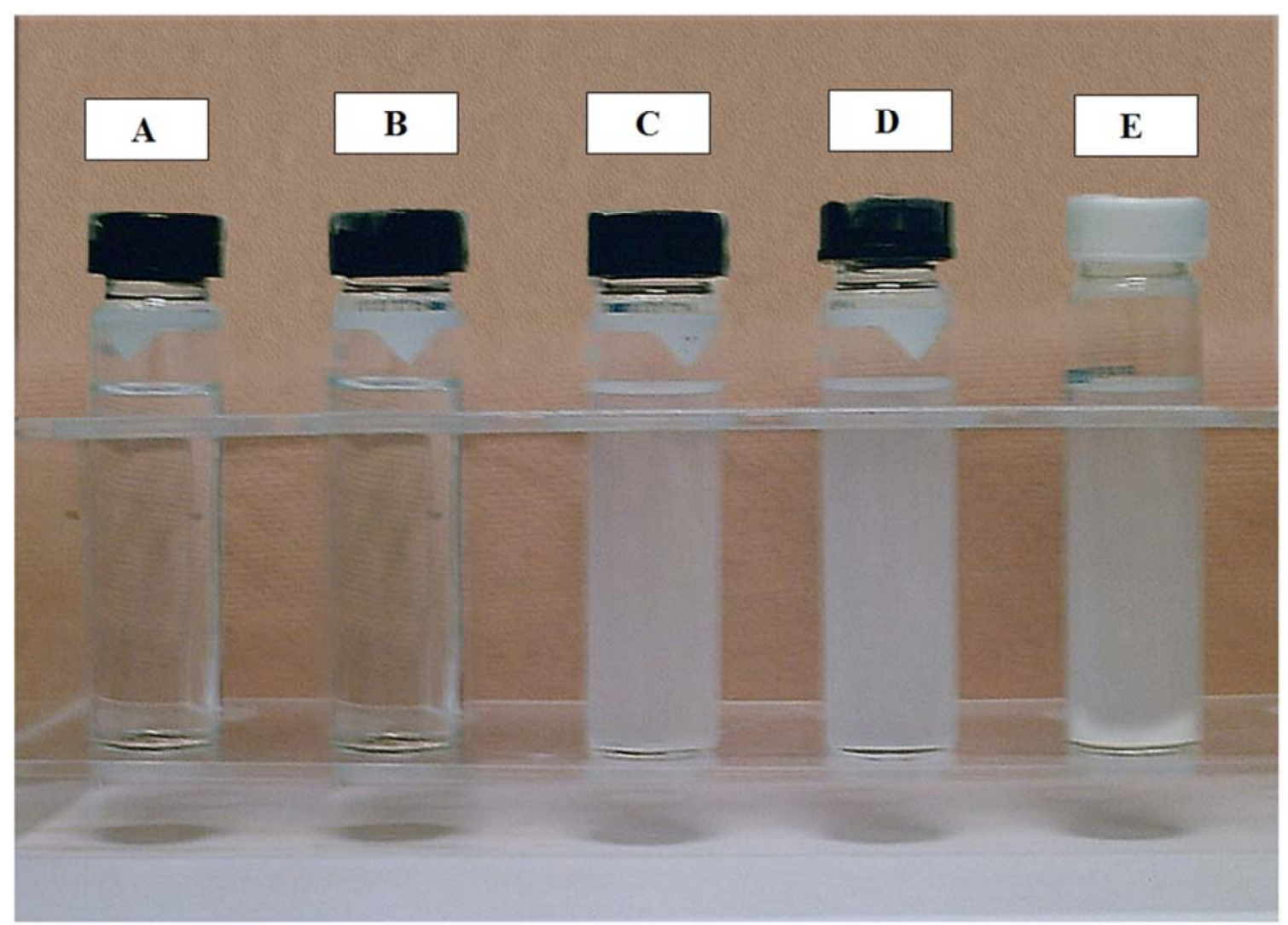

Figure 2. Visual turbidity in $100 \mathrm{mM} \mathrm{NaNO} \mathrm{N}_{3}$ and $\mathrm{pH} 7$ for (A) $2 \mathrm{~g} / \mathrm{L} C M D$ in absence of metal, (B) $2 \mathrm{~g} / \mathrm{L} C M D$ in presence of Ca ${ }^{2+}$ with $M / P=1$, (C) $C M D 2$ $\mathrm{g} / \mathrm{L}$ in presence of $C d^{2+}$ with $M / P=1$, (D) $2 \mathrm{~g} / \mathrm{L} C M D$ in presence of $C d^{2+}$ with $M / P=1.8$, (D) $2 \mathrm{~g} / L C M D$ in presence of $C d^{2+}$ with $M / P=2$.

The photography of the associated CMD solutions on Figure 2 (C, D and E) shows relatively high turbid solutions for these polysaccharide/cadmium mixtures, reflecting experimental data that are up to 500 times more higher than those of polysaccharides/calcium mixtures. Such behavior is characteristic of high interactions between CMD macromolecules and cadmium, previously [49] seen at molecular state. The shift of the plots toward low metal/polymer ratios as CMD concentration increase may be due to a solvent effect. It is probable that with the increase of polysaccharide concentration, the polymer-polymer interactions induce a chain reconformation which favors intermolecular interactions arising from the bridge between carboxylate and cadmium ions [49]. In this way, these intermolecular interactions can lead to complexes formation that increase with large metal/polymer ratios. The presence of maximums on the plots correspond to critical coagulation ratios. There are found at almost a same peak height which occurs for metallic ion at an identical critical aggregation concentration of $7.5 \mathrm{mM}$, indicating a limit size of the aggregates. In the domain preceding these peaks, the turbidity describes a plateau for which data are proportional to CMD concentrations. It corresponds to an area below which no aggregation can be detected. Such a feature may be mainly attributed to intramolecular interactions occurring between cadmium and carboxylate ions $[49,50]$. Harnsilawat and al. [51] that observed similar interactions between polysaccharides and proteins have attributed such behavior to the formation of insoluble complexes coming from the electrostatic attraction between negatively charged alginate and positive charge protein. Above the intramolecular interactions domain, any increase of metal/polymer ratio leads to sharp increase in turbidity. The addition of metal favors the intermolecular interactions between polymer and metal. Its gradual increase up to the peaks indicates that the diffusion of the objects in suspension is proportional to their number and varies like the square volume. The increase of turbidity values also suggest that stable and probably compact aggregates begin to form with apparent density very close to 1 . These aggregates, arising from intermolecular interactions, are distributed throughout the suspension, since in spite of the turbidity increasing, the suspension remains stable. Beyond the peaks, turbidity begins to decrease progressively with molar ratios meaning that an additional supply of cadmium amplifies at the same time intermolecular bridges and destabilizes aggregates which likely become more compact. The turbidity decreases progressively and falls down below that one originally observed, indicating that the colloidal suspension has completely decanted. Phase separation is then observed with the formation of a sediment (Figure 2(E)).

\subsection{Kinetics of Aggregates Growth}

The analysis of turbidimetric plots on the interactions 
between CMD macromolecules and cadmium shows that the aggregation conditions are defined for the ratio values greater than 2, 1 and 0.5 for respective polymer concentrations of 0.5 $\mathrm{g} / \mathrm{L}, 1 \mathrm{~g} / \mathrm{L}$ and $2 \mathrm{~g} / \mathrm{L} \mathrm{CMD} \mathrm{as} \mathrm{summarized} \mathrm{in} \mathrm{the} \mathrm{phase}$ diagram of CMD in Figure 3.

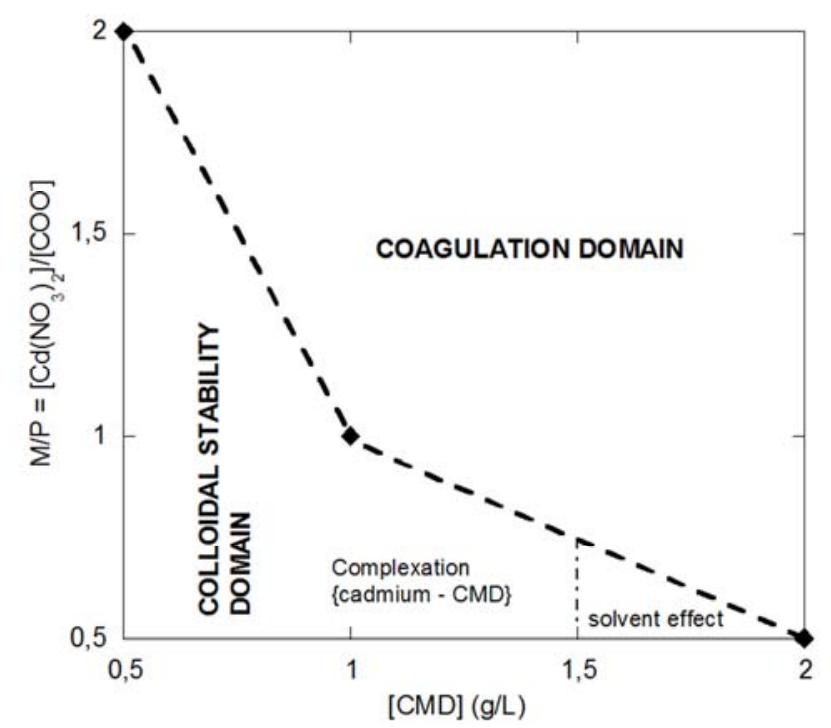

Figure 3. Phase diagram of $C M D-C d$ system as a function of polymer concentration.

In order to characterize the coagulation process of CMD macromolecules, we studied their kinetics knowing that this feature is determined by the nature of the interactions (more or less attractive), the mobility of objects (relative to their size), and the structure of the aggregates (fractal dimension). In taking account the turbidimetric results, the aggregation kinetic is monitored for a CMD concentration of $0.5 \mathrm{~g} / \mathrm{L}$ in the domains corresponding to the region preceding the peak $(\mathrm{M} / \mathrm{P}=2.5)$, then at the peak maximum $(\mathrm{M} / \mathrm{P}=3.5)$ and finally to the area beyond the peak $(\mathrm{M} / \mathrm{P}=5)$.

This analysis was followed by flocs size measurements, which represent an important parameter that provides informations on the evolution of colloidal aggregates. This feature as a function of time is illustrated on Figure 4A and Figure 4B. As can be seen, the plots show clearly an increase in the size of the objects with an initial velocity (Figure 5) and a maximum size (Figure 6) that are functions of the metal concentration added to the suspension. Such variations indicate that the metal plays a catalytic role on the aggregation process of the polymer. A closer inspection of the curves in Figsures $4 \mathrm{~A}$ and $4 \mathrm{~B}$ shows that the aggregation process is governed by different behaviors on all examined $\mathrm{M} / \mathrm{P}$ ratios. At first, the aggregation rates increase up to a critical and limit aggregate size which then stabilizes with time for the $\mathrm{M} / \mathrm{P}$ ratio of 5 , but decreases for $\mathrm{M} / \mathrm{P}$ ratios of 2.5 and 3.5 at sufficiently long time.

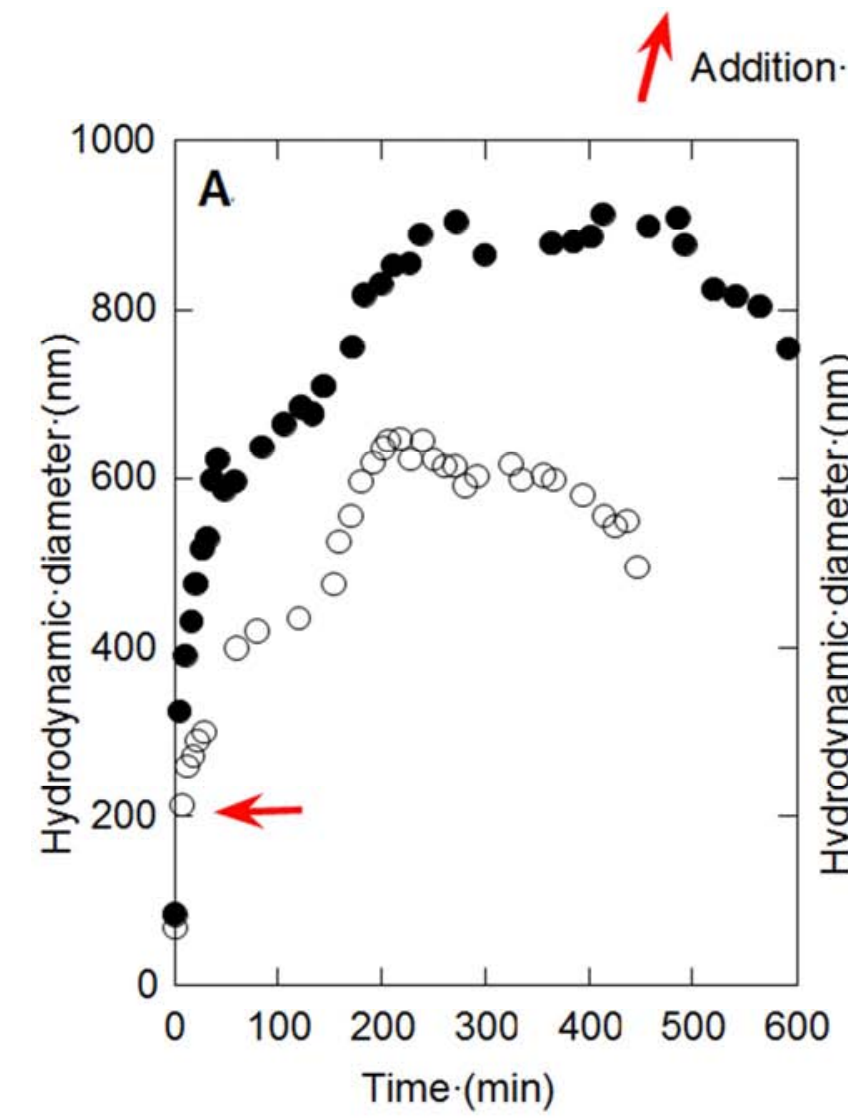

of metal. to 


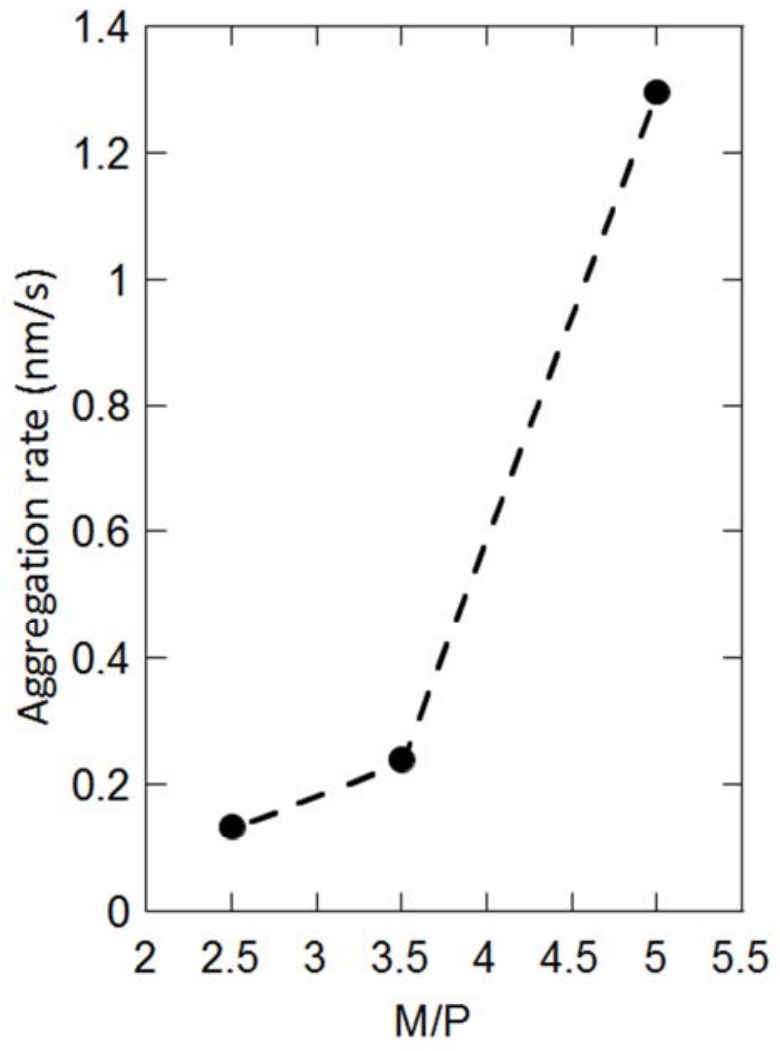

Figure 5. Initial velocity of aggregation kinetics as a function of metal to polymer ratios (evaluated from Figure 4.).

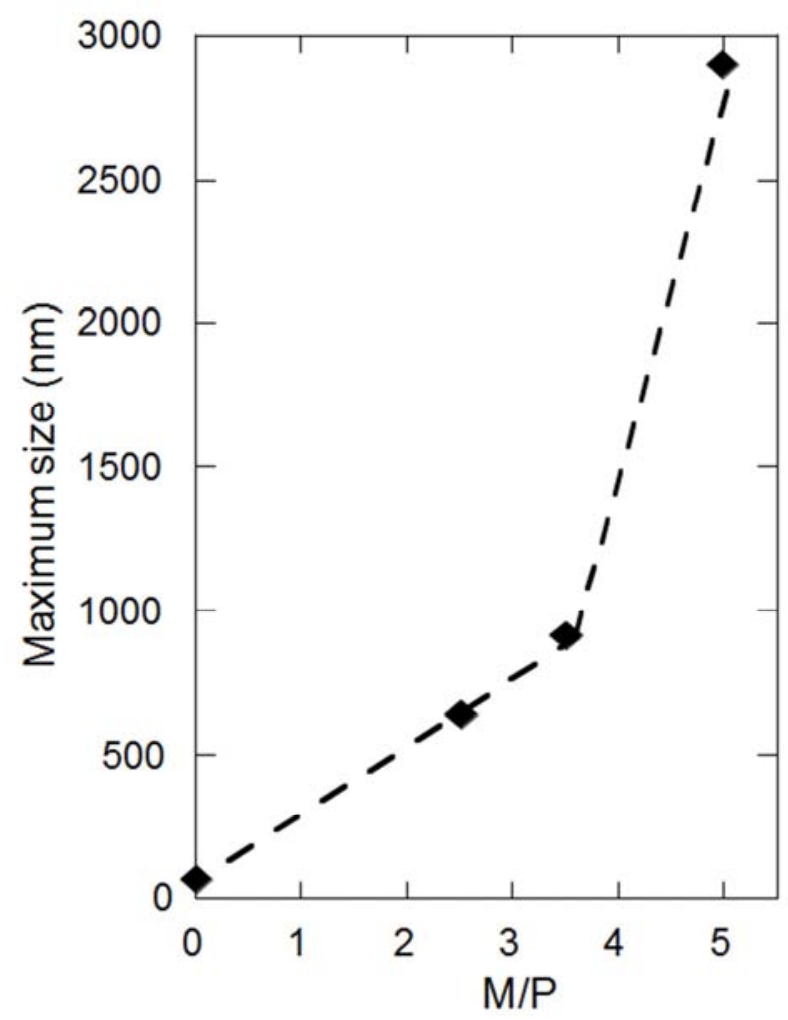

Figure 6. Maximum size as a function of metal to polymer ratios (deduced from Figure 4.).
It appears that the phase of aggregate growth occurs for $\mathrm{M} / \mathrm{P}$ ratio of 2.5 according to an aggregation rate characterized by a shoulder. This phenomenon may be related to a problem of solution mixing. Indeed, after addition of metal to the polymer suspension, the solution is not stirred. It is so possible that when the metal is injected at low concentrations, it only interact beforehand with the first macromolecules within its neighbor environment according to a rate corresponding to the initial velocity. In a second step, as the metal ions are homogeneously distributed throughout the suspension, they interact with the other macromolecules, resulting in a further increase in the velocity of aggregation. An increase in the $\mathrm{M} / \mathrm{P}$ ratio corresponding to metal content increasing allows to overcome both of these steps, as shown by the growth kinetics at the $\mathrm{M} / \mathrm{P}$ ratios of 3.5 and 5. Due to the macromolecular charges screening at ionic strength of $100 \mathrm{mM} \mathrm{NaNO}_{3}$, repulsions between electrical doubles layers are negligible. Under such conditions, the mechanism of CMD coagulation is a diffusion-limited aggregation insofar the particles have no longer or may be any energy barrier to cross before they interact. This assumption is experimentally supported by the shape of aggregation kinetics since the rate of coagulation decreases as a function of time [52] in particular for the $\mathrm{M} / \mathrm{P}=2.5$ and $\mathrm{M} / \mathrm{P}=3.5$. In addition, relatively high velocities and large aggregate sizes are the signature of rapid aggregation, which are induced by attractive interactions arising from Van Der Waals interactions. The associated conformations derive from the effective kind of the shocks induced by each of the collisions between particles. In their growing phase, the aggregates are stable in suspension and little compact. The obtained maximum sizes observed indicate that the objects reached their maximum threshold of compactness, their collision with other aggregates or adjacent particles being enough to destabilize them. The low decrease of aggregation rates at relatively long times indicates that sedimentation may be slow due probably to aggregate restructuring. On turbidimetric measurements, the mechanical stirring modified the state of the system since it was observed that after 2 hours of mixing and 17 hours at rest, the suspension is always stable at the $\mathrm{M} / \mathrm{P}$ ratios of 2.5 and 3,5 . The situation is different for suspension at $\mathrm{M} / \mathrm{P}$ of 5 for which, the maximum size is already reached after about $40 \mathrm{~min}$. Despite its value up to 4 times higher than that obtained for $\mathrm{M} / \mathrm{P}$ ratios of 2.5 and 3.5, the aggregates remain in suspension while the turbidity measurements showed that at this ratio the aggregates sediment. It seems that this process may be slower under the conditions of aggregation kinetics of ratio $\mathrm{M} / \mathrm{P}$ of 5.

\section{Conclusion}

The present study allowed to examine the aggregation process of soft particle as experimental data to complete few works in literature on soft materials. At that end, the 
aggregation kinetics of carboxymethyldextran, a soft and anionic polysaccharide induced by $\mathrm{Cd}^{2+}$, were analyzed in aqueous $\mathrm{NaNO}_{3}$ solutions through turbidimetric and dynamic light measurements. Turbidimetry is firstly used to point out the destabilization conditions of the colloidal dispersion and as second step, the characterization of the kinetics and aggregates are performed by dynamic light scattering technique. Results show that aggregation behavior of the suspension is modulated by the metal to polymer molar concentrations ratios. For each examined polysaccharide, a critical coagulation value for the metal to polymer ratio is found. Below this ratio, the colloidal dispersion is at first stable due to the intramolecular interactions polymer/cadmium that govern and then begin to coagulate sharply as a result of intermolecular interactions. The aggregation process is further increased above critical ratio value leading a destabilization of the suspension for sufficiently high ratios. The rate of aggregation and the size evolution indicate a diffusion-limited aggregation, which is more pronounced as the metal to polymer concentration ratio is increased. The turbidity and the dynamic light scattering are simple and efficient approach that allow us to provide the destabilization conditions of soft particle suspension and to analyse their kinetics aggregation. The characteristics of these aggregate can be further investigated by other techniques like static light scattering in order to determinate exactly features as aggregates fractal dimension.

\section{References}

[1] N. M Kovalchuk, V. M Starov, "Aggregation in colloidal suspensions: Effect of colloidal forces and hydrodynamic interactions," Adv. Colloid Interface Sci., Vol. 179-182, pp. 99-106, 2012.

[2] H. Wu, M. Lattuada M. Morbidelli, "Dependence of fractal dimension of DLCA clusters on size of primary particles," Adv. Colloid Interface Sci., Vol. 195-196, pp. 41-49, 2013.

[3] B. R. Saunders, M. L. Turner, "Nanoparticle-polymer photovoltaic cells," Adv. Colloid Interface Sci., Vol.138, pp.1-23, 2008.

[4] B. G. Prevo, E. W. Hon, O. D. Velev, "Assembly and characterization of colloid-based antireflective coatings on multicrystalline silicon solar cells," J. Mater. Chem., Vol. 17, pp.791-799, 2007.

[5] H. Zhang, X. Yu, P. V. Braun, "Three-dimensional bicontinuous ultrafast-charge and -discharge bulk battery electrodes." Nat. Nanotechnol., Vol. 6, pp. 277-281, 2011.

[6] X-F. Tang, Z-G. Yang, W-J. Wang, “A simple way of preparing high-concentration and high-purity nano copper colloid for conductive ink in inkjet printing technology," Colloid Surf. A Physicochem. Eng. Asp., Vol. 360, pp. 99-104, 2010.

[7] A. L. Rogach, A. Eychmüller, S. G. Hickey, S.V. Kershaw, "Infrared-emitting colloidal nanocrystals: synthesis, assembly, spectroscopy, and applications," Small, Vol. 3, pp. 536-557, 2007.

[8] D. P. Landau, F. Family, Kinetics of Aggregation and
Gelation, $1^{\text {st }}$ ed, Amsterdam, eds. North Holland, Netherlands, 1984.

[9] H. E. Stanley, N. Ostrowsky, Eds, On Growth and Form, $1^{\text {st }}$ ed., Vol.100, eds. Springer Netherlands: Netherlands, 1986.

[10] R. Pynn, A. Skjeltorp, Eds; Scaling Phenomena in Disordered Systems Plenum, Eds. NewYork, 1986.

[11] M. Carpineti, M. Giglio, “Aggregation phenomena," Adv. Colloid Interface Sci., Vol. 46, pp. 73-79, 1993.

[12] M. Hütter, "Local Structure Evolution in Particle Network Formation Studied by Brownian Dynamics Simulation," J. Colloid Interface Sci., Vol. 231 (2), pp. 337-350, 2000.

[13] J. Cordelair, P. Greil, "Flocculation and coagulation kinetics of $\mathrm{Al}_{2} \mathrm{O}_{3}$ Suspensions," J. Eur. Ceram. Soc., Vol. 24 (9), pp. 2717-2722, 2004.

[14] B. V. R. Tata, M. Rajalakshmi, A. K. Arora, "Vapor-liquid condensation in charged colloidal suspensions," Phys. Rev. Lett., Vol. 69, pp. 3778-3781, 1992.

[15] A. Videcoq,, M. Han, P. Abelard, C. Pagnoux, F. Rossignol, R. Ferrando, "Influence of the potential range on the aggregation of colloidal particles," Physica A, Vol. 374, pp. 507-516, 2007.

[16] F. Gambinossi, S. E. Mylon, J. K. Ferri, "Aggregation kinetics and colloidal stability of functionalized nanoparticles," Adv. Colloid Interface Sci., Vol. 222, 332-349, 2015.

[17] M. Y. Lin, R. Klein, H. M. Lindsay, D. A. Weitz, R. C.Ball and P. Meakin, J, "The structure of fractal colloidal aggregates of finite extent," Colloid Interface Sci., Vol. 137, pp. 263-280, 1990.

[18] J. E. Martin, Judy Odinek, "On the Turbidity of Silica Gels: aggregation in the presence of coulomb interactions," J. Colloid Interface Sci., Vol. 154 (2), pp. 461-480, 1992.

[19] P. Bezot, C. Hesse-Bezot, C. Diraison, "Aggregation kinetics of colloidal suspensions of engine soots. Influence of polymeric lubricant additives," Carbon Vol. 35, No. 1, pp. 53-60, 1997.

[20] Kathrin Harre, Gerhard Wegner, "Solution properties and kinetics of aggregation of an alkyl-substituted poly (pphenylene)," Polymer, Vol. 47, pp. 7312-7317, 2006.

[21] A. Fernandez-Nieves, A. Fernandez-Barbero, B. Vincent, F. J. de las Nieves, "Reversible aggregation of soft particles," Langmuir Vol. 17, pp. 1841-1846, 2001.

[22] H. Cheng, C. Wu, M. A. Winnik, "Kinetics of reversible aggregation of soft polymeric particles in dilute dispersion," Macromolecules, Vol 37 (13), pp. 5127-5129, 2004.

[23] S. Roldán-Vargas, A. Martín-Molina, M. Quesada-Pérez, R. Barnadas-Rodríguez, J. Estelrich, J. Callejas-Fernández, "Aggregation of liposomes induced by calcium: A structural and kinetic study", Phys. Rev. E, Vol. 75, 021912, 2007.

[24] S. Roldán-Vargas, R. Barnadas-Rodríguez, A. Martín-Molina, M. Quesada-Pérez, J. Estelrich, J. Callejas-Fernández, "Growth of lipid vesicle structures: From surface fractals to mass fractals," Phys. Rev. E, Vol.78, 010902, 2008.

[25] S. K. Filippov, A. V. Lezov, O. Y. Sergeeva, A. S. Olifirenko, S. B. Lesnichin, N. S. Domnina, E. A. Komarova, M. Almgren, G. Karlsson, P. Štepanek, "Aggregation of dextran hydrophobically modified by sterically-hindered phenols in aqueous solutions: Aggregates vs. single molecules," Eur Polym J., Vol. 44, pp. 3361-3369, 2008. 
[26] M. H. Shen, Y. G. Yin, A. Booth, J. F. Liu, "Effects of molecular weight-dependent physicochemical heterogeneity of natural organic matter on the aggregation of fullerene nanoparticles in mono- and di-valent electrolyte solutions," Water Res., Vol. 71, pp. 11-20, 2015.

[27] P. Meakin, "Formation of Fractal Clusters and Networks by Irreversible Diffusion-Limited Aggregation,” Phys. Rev. Lett. Vol. 51 (13), pp. 1119-1122, 1983.

[28] M. Kolb, R. Botet, R. Jullien, "Scaling of Kinetically Growing Clusters,” Phys. Rev. Lett., Vol. 51, pp.1123-1126, 1983.

[29] P. Meakin, "Fractal aggregates," Adv. Colloid Interface Sci., Vol. 28, pp. 249-331, 1988.

[30] P. Meakin, "Simulation of the Kinetics of Aggregation: Fractals and Scaling," Croat. Chem. Acta, 65 (2) pp. 237-267, 1992.

[31] P. Meakin, "Aggregation kinetics," Phys. Scripta, Vol. 46, pp. 295-331, 1992.

[32] R. Jullien, "The Application of Fractals to Colloidal Aggregation," Croat. Chem. Acta, Vol. 65 (2) pp. 215-235, 1992.

[33] M. Lattuada, P. Sandkühler, H. Wu, J. Sefcik, M. Morbidelli, "Aggregation kinetics of polymer colloids in reaction limited regime: experiments and simulations," Adv. Colloid Interface Sci., Vol. 103, pp. 33-56, 2003.

[34] M. Lattuada, H. Wu, P. Sandkuhler, J. Sefcik, M. Morbidelli, "Modelling of aggregation kinetics of colloidal systems and its validation by light scattering measurements", Chem. Eng. Sci., Vol. 59, pp. 1783-1798, 2004.

[35] T. A. Witten, L. M. Sander, "Diffusion-Limited Aggregation, a Kinetic Critical Phenomenon," Phys. Rev. Lett., Vol 47 (19), pp. 1400-1402, 1981.

[36] T. A. Witten, L. M. Sander, "Diffusion-limited aggregation," Phys. Rev. B Vol. 27, pp. 5686-5697, 1983.

[37] R. C. Ball, R. M. Brady, G. Rossi, B. R. Thompson, "Anisotropy and Cluster Growth by diffusion-limited aggregation,” Phys. Rev. Lett., Vol. 55 (13), pp. 1406-1409, 1985.

[38] R. Jullien, M. Kolb, "Hierarchical model for chemically limited cluster-cluster aggregation,” J. Phys. A: Math. Gen., Vol. 17 (12), L639, 1984.

[39] W. D. Brown, R. C. Ball, "Computer simulation of chemically limited aggregation,” J. Phys. A, Vol. 18 (9) L517, 1985.

[40] R. C. Ball, D. A. Weitz, T. A. Witten, F. Leyvraz, “Universal kinetics in reaction-limited aggregation," Phys. Rev. Lett., Vol. 58, pp. 274-277, 1987.

[41] P. Arosio, "Stability and aggregation kinetics of colloidal systems: Application to polymer colloids, proteins and peptides," Swiss Federal Institute of Technology, Thesis, Zurich, 2011.

[42] N. Akkaya, A. T. Bilgiçli, A. Aytekin, M. N. Yarasir, M. Kandaz, "Novel metal (III) and metal free soft phthalocyanine metal ion sensors bearing (1-hydroxyhexan-3-ylthio)substituents: Synthesis, characterization, aggregation behavior," Polyhedron, Vol. 85, pp. 857-863, 2015.

[43] S. Roldán-Vargas, R. Barnadas-Rodríguez, M. Quesada-Pérez, J. Estelrich, J. Callejas-Fernández, "Surface fractals in liposome aggregation,” Phys. Rev. E Vol. 79, 011905, 2009.

[44] F. Chaubet, J. Champion, O. Maïga, S. Mauray, J. Jozefonvicz, "Synthesis and structure anticoagulant property relationships of functionalized dextrans: CMDBS," Carbohydr. Polym., Vol. 28, pp. 145-152, 1995.

[45] M. Mauzac, J. Josefonvicz, "Anticoagulant activity of dextran derivatives. Part I: Synthesis and characterization," Biomaterials, Vol. 5, pp. 301-304, 1984.

[46] J. F. L. Duval, H. P. van Leeuwen, "Electrokinetics of diffuse soft interfaces. 1. Limit of low donnan potentials," Langmuir, Vol. 20, pp. 10324-10336, 2004.

[47] J. F. L. Duval, "Electrokinetics of diffuse soft interfaces. 2. Analysis based on the nonlinear Poisson-Boltzmann equation," Langmuir, Vol. 21, pp. 3247-3258, 2005.

[48] J. F. L. Duval, H. Ohshima, "Electrophoresis of diffuse soft particles," Langmuir, Vol. 22, pp. 3533-3546, 2006.

[49] J. P. S. Sagou, E. Rotureau, F. Thomas, J. F. L. Duval, "Impact of metallic ions on electrohydrodynamics of soft colloidal polysaccharides," Colloids Surf. A Vol. 435, pp. 16-21, 2013.

[50] J-P. S. Sagou, S. S. Kouassi, J. Y. Y. Andji, "Influence of polyeclectrolyte concentration and metallic ions on viscosity of soft colloidal polysaccharides," Int. J. Aca. Sci. Res., Vol. 4, pp. 75-82, 2016.

[51] T. Harnsilawat, R. Pongsawatmanit, D. J. McClements, "Characterization of b-lactoglobulin-sodium alginate interactions in aqueous solutions: a calorimetry, light scattering, electrophoretic mobility and solubility study," Food Hydrocoll., Vol. 20, pp. 577-585, 2006.

[52] H. Shui, H. Zhou, "Kinetic study on the aggregation of coal soluble constituents in Solution," Fuel Process. Technol., Vol. 86, pp. 661-671, 2005. 